Article

\title{
Prevalence of Meibomian Gland Dysfunction and Its Effect on Quality of Life and Ocular Discomfort in Patients with Prosthetic Eyes
}

\author{
Alessandro Meduri ${ }^{1}{ }^{*}$, Rino Frisina ${ }^{2}$, Miguel Rechichi ${ }^{3}$ and Giovanni William Oliverio ${ }^{1}$ \\ 1 BIOMORF Deperatement, Ophthalmology Clinici, University of Messina, 98124 Messina, Italy; \\ g.w89@me.com \\ 2 Department of Ophthalmology, University of Padua, 35121 Padua, Italy; frisinarino@unipd.com \\ 3 Centro Polispecialistico Mediterraneo, 88050 Sellia Marina, Italy; miguel.rechichi@libero.it \\ * Correspondence: ameduri@unime.it
}

Received: 23 April 2020; Accepted: 8 June 2020; Published: 9 June 2020

\begin{abstract}
Purpose: To evaluate the influence of ocular discomfort and meibomian gland dysfunction (MGD) on quality of life in patients with an ocular prosthesis. Methods: a prospective analysis was conducted on 18 patients with a unilateral ocular prosthesis. Evaluation of ocular discomfort symptoms, lid margin abnormalities (LMA), meibomian gland expression, meibography and a psychometric evaluation using the National Eye Institute Visual Function Questionnaire (NEI VFQ), Facial Appearance subscale of the Negative Physical Self Scale (NPSS-F), Hospital Anxiety and Depression Scale (HADS) and the DAS24 to evaluate anxiety and depression. Results: the statistically significant differences observed between normal and prosthetic eyes related to ocular symptoms and the meibography score $(p=0.0003)$. A negative correlation was reported between NEI VFQ score and meibography score $(\mathrm{r}=-0.509 ; p$-value $=0.022)$. A positive correlation was detected with NPSS $(\mathrm{r}=0.75 ; p$-value $<0.0001)$, anxiety HADS score $(\mathrm{r}=0.912 ; p$-value $=0.001)$ and depression HADS score $(r=0.870 ; p$-value $>0.0001)$. Conclusion: MGD represents the most common cause of evaporative dry eye disease, due to the reduction of the thickness of the lipid layer of the tear film. The occurrence of MGD in patients with prosthetic eyes is very common. Anxiety and depression were correlated to ocular discomfort and MGD, and this could affect the quality of life in patients with an ocular prosthesis.
\end{abstract}

Keywords: ocular prosthesis; meibomian gland dysfunction; quality of life; ocular discomfort

\section{Introduction}

The loss of an eye presents severe emotional stress and commonly results in negative consequences to social behavior. Patients with an ocular prosthesis reported a high level of anxiety and depression as a consequence of their changed appearance. In this sense, the use of an ocular prosthesis purposes to improve cosmetic appearance, as well as ameliorating social acceptance [1]. Nowadays, ocular prosthesis can be stock or patient-customized, considering several factors, such as the anatomy and tissue bed of the socket, and individualized aesthetic requirements. The main material used for the fabrication of ocular prostheses is polymethyl methacrylate (PMMA), which is compatible with tissues, and has easy color modification abilities and elevated aesthetic appearance. Numerous techniques to improve the aesthetic results of the ocular prosthesis have been proposed, such as painting the sclera and iris, the use of transparent grids for proper orientation and the use of a digital image of the normal eye [2]. The principal causes for the need of an ocular prosthesis comprise tumors, congenital defects and malformations, irreparable trauma, end-stage eye diseases, and severe ocular diseases associated with uncontrolled pain, such as neovascular glaucoma, or an unattractive appearance, such 
as phthisis bulbi [3,4]. Eye removal surgery is classified into evisceration, enucleation and exenteration. Evisceration includes the removal of the ocular contents, leaving in place the sclera shell. The mobility of the eviscerated globe implant is preserved, since the extraocular muscles are intact. Enucleation consists in the removal of the eyeball after the extraocular muscles and the optic nerve have been separated. Sufficient space is formed for the prosthesis, with movement of the fornix within the enucleated socket providing mobility to the ocular prosthesis. Exenteration is the surgical removal of the complete contents of the orbit. The eyelids may or may not be involved. Exenteration defects in some instances may be allowed to heal by secondary intent, but adequate space must remain in the resultant defect to allow the prosthesis to be positioned superiorly and posteriorly enough for a good cosmetic appearance [5]. An orbital implant is used to fill the orbital cavity or the scleral shell, and to replace the consequent volume reduction due to the removal of the eye. The eye's motility is preserved, as the scleral implant is attached to the ocular muscles. Several types of implants exist, differing in shape (spherical or oval), being stock or customized, porous or non-porous, and in the presence of a peg or motility post [4,5]. Chronic ocular discomfort represents one of the most common adverse events referred to in long-standing patients with an ocular prosthesis. Many patients with an ocular prosthesis report varying degrees of ocular discomfort, such as discharge, dryness, irritation and a sticky sensation. Numerous mechanisms that could lead to ocular discomfort in prosthetic eye wearers were suggested, such as the infection of the anophthalmic socket, glutinous surface deposits and a roughened prosthesis [6,7]. A recent study analyzed the role of the tear film and demonstrated the linear correlation between tear deficiency and discomfort reported in patients with an ocular prosthesis [7]. Recent studies also confirmed the relationship between MGD and contact lens wear, as well as in ocular prosthesis wear, demonstrating a larger grade of Meibomian gland loss and alterations compared with normal paired eyelids [8,9]. Meibomian gland dysfunction (MGD) represents the most common cause of evaporative dry eye disease, due to the reduction of the thickness of the lipid layer of the tear film [10]. The aim of this study is to evaluate the prevalence of MGD in patients with ocular prostheses reporting symptoms of ocular discomfort, and their impact on quality of life.

\section{Results}

Demographic data is reported in Table 1. Eighteen patients of mean age $51.3 \pm 16.8$ years and mean length of prosthesis duration $13.6 \pm 5.1$ years were studied. The most frequent causes of blindness were trauma (ten patients), cancer (five patients) and other ocular diseases (three patients) such as endophthalmitis and one case of neo-vascular glaucoma. A case of unilateral ocular prosthesis due to neo-vascular glaucoma is reported in Figure 1. Evisceration was the most common surgery (10 patients). All ocular prostheses were polymethyl methacrylate (PMMA). Sixteen patients had a customized prosthesis, presenting a good aesthetic appearance. Only two patients had a stock prosthesis. In Table 2 summarises scores regarding ocular symptoms, lid margin abnormalities (LMA), meibomian gland expression and meiboscore related to the normal eye and the prosthetic eye in the upper and lower eyelid. Significant statistical differences were noted between the normal eye and prosthetic eye related to ocular symptoms ( $p$-value $<0.0001)$, LMA $(p=0.0006)$, meibomian gland expression score $(p=0.0003)$, and in the meiboscore of the upper eyelid $(p=0.0004)$, lower eyelid $(p=0.0003)$ and total meiboscore $(p=0.0003)$. A positive correlation between duration of prosthesis and meiboscore in the prosthetic eye $r=0.878 ; p<0.00195 \%$ CI: $[0.688,0.956]$ was observed. All psychometric data are reported in Table 3. A negative correlation was reported between NEI VFQ score and meiboscore $(\mathrm{r}=-0.509 ; p$-value $=0.022)$. Positive correlations were detected with NPSS $(\mathrm{r}=0.75 ; p$-value $<0.0001)$; anxiety HADS score $(\mathrm{r}=0.912 ; p$-value $<0.001)$; depression HADS score $(\mathrm{r}=0.870 ; p$-value $<0.0001)$; DES24 ( $\mathrm{r}=0.686 ; p$-value $<0.0001)$ and VAS score for sadness $(\mathrm{r}=0.657 ; p$-value $=0.002)$ anger $(\mathrm{r}=0.741 ; p$-value $<0.0001)$ and shamefulness $(\mathrm{r}=0.744 ; p$-value $<0.0001)$. (Figure 2 and Table 4$)$. 
Table 1. Clinical Characteristics of the Study Population.

\begin{tabular}{cc}
\hline Parameters & Value \\
\hline Total patients & 18 \\
Age (years) & $51.3 \pm 16.8$ \\
Gender & $10(55.6 \%)$ \\
Male & $8(44.4 \%)$ \\
Female & $13.6 \pm 5.1$ \\
Duration of prosthesis (years) & $10(55.6 \%)$ \\
Cause of eye loss & $5(27.8 \%)$ \\
Trauma & $3(16.6 \%)$ \\
Cancer & \\
Further disease & $10(55.6 \%)$ \\
Surgery & $8(44.4 \%)$ \\
Evisceration &
\end{tabular}

Data are presented as $\mathrm{n}(\%)$, and mean \pm standard deviation.

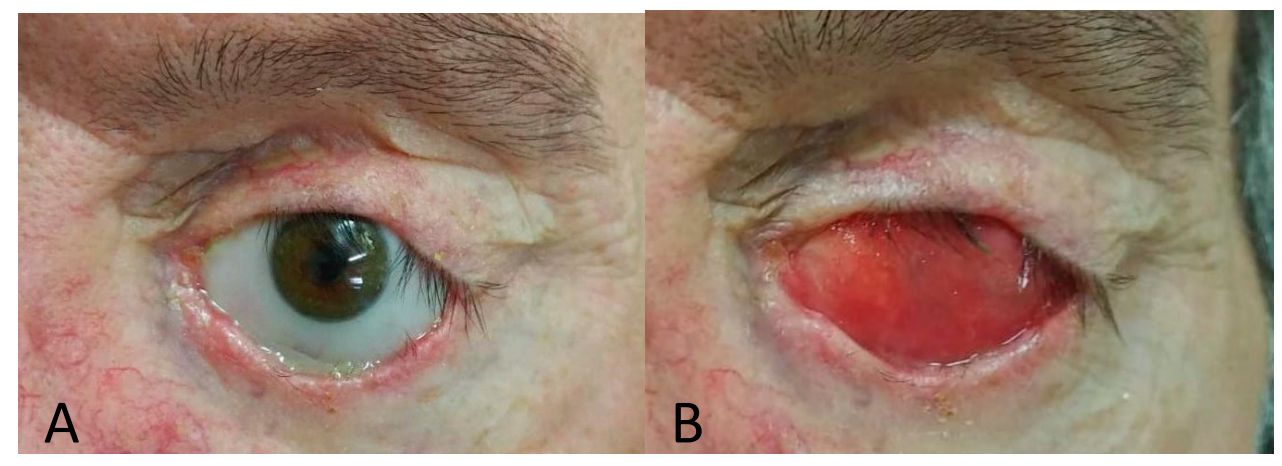

Figure 1. Ocular prosthesis (A), anophthalmic socket (B).

Table 2. Comparison of meibomian gland and lid margin evaluation in prosthesis and normal eye.

\begin{tabular}{cccc}
\hline & $\begin{array}{c}\text { Prosthetic Eye } \\
(\text { Mean } \pm \text { SD) }\end{array}$ & $\begin{array}{c}\text { Normal Eye } \\
(\text { Mean } \pm \text { SD) }\end{array}$ & $p$-Value * \\
\hline Symptoms score & $8.8 \pm 2.8$ & $2.5 \pm 1.7$ & $<0.0001$ \\
Lid margin abnormalities & $2.1 \pm 0.7$ & $0.4 \pm 0.6$ & 0.0006 \\
Meibomian gland expression & $2.5 \pm 0.6$ & $0.4 \pm 0.5$ & 0.0003 \\
Meiboscore & & & \\
Upper eyelid & $2 \pm 0.7$ & $0.4 \pm 0.5$ & 0.0004 \\
Lower eyelid & $1.3 \pm 0.5$ & $0.2 \pm 0.4$ & 0.0003 \\
Complessive & $3.1 \pm 0.78$ & $0.41 \pm 0.5$ & 0.0003 \\
\hline
\end{tabular}

All data are reported as mean \pm standard deviation. ${ }^{*}$ Wilcoxon signed-rank test. Bold characters for $p$-value $<0.05$.

Table 3. Psychometric data.

\begin{tabular}{cc}
\hline & $\begin{array}{c}\text { Score } \\
\text { (Mean } \pm \text { SD) }\end{array}$ \\
\hline Vision-specific composite & $38.4 \pm 16.9$ \\
Appearance concern & $2.8 \pm 1.13$ \\
Shame & $4.94 \pm 2.33$ \\
Sadness & $5.35 \pm 2.87$ \\
Anger & $5.52 \pm 2.15$ \\
Anxiety & $6.76 \pm 2.33$ \\
Depression & $6.29 \pm 2.77$ \\
DAS24 & $45.88 \pm 25.63$ \\
\hline
\end{tabular}

Vision-specific composite refers to NEI VFQ score; appearance concern refers to NPSS score; shame, sadness and anger were evaluated using a visual analogue score. All data are reported as mean \pm standard deviation. 

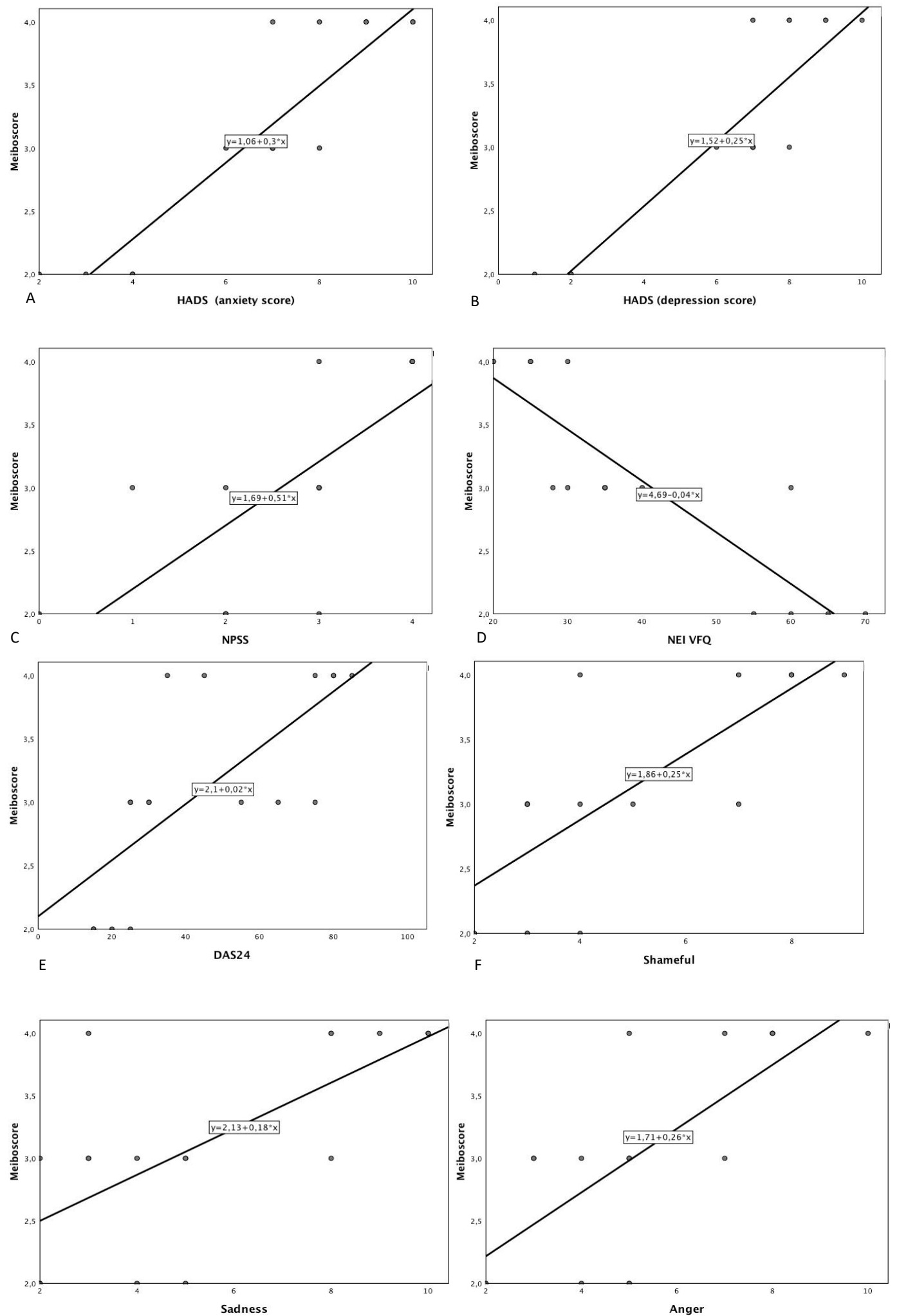

H

Figure 2. Scatter plot showing the relationship between total meiboscore (total) and (A) Hospital Anxiety and Depression Scale (anxiety score); (B) Hospital Anxiety and Depression Scale (depression score); (C) Negative Physical Self Scale-Facial appearance subscale score; (D) National Eye Institute Visual Function Questionnaire; (E) Derriford Appearance Scale; (F) Shameful (visual analogue scale); (G) Sadness (visual analogue scale); (H) Anger (visual analogue scale). 
Table 4. Pearson's correlation between Meiboscore (total) and psychometric score.

\begin{tabular}{ccc}
\hline & $\mathbf{r}$ & $p$-Value \\
\hline NEI VFQ & -0.884 & $<0.001$ \\
NPSS & 0.732 & $<0.001$ \\
HADS (Anxiety) & 0.912 & $<0.001$ \\
HADS (Depression) & 0.870 & $<0.001$ \\
DAS24 & 0.686 & $<0.001$ \\
Shameful & 0.744 & $<0.001$ \\
Sadness & 0.657 & 0.002 \\
Anger & 0.741 & $<0.001$
\end{tabular}

Legend: NEI VFQ-The National Eye Institute Visual Function Questionnaire; NPSS-Negative Physical Self Scale; HADS—Hospital Anxiety and Depression Scale; DAS-24—Derriford Appearance Scale.* ANOVA test, bold characters for $p$-value $<0.05$.

\section{Discussion}

Chronic ocular discomfort represents one of the most common adverse events referred to in long-standing patients with an ocular prosthesis. Chronic discharge, in patients with an ocular prosthesis, could be related to several conditions. In particular, they could be classified as prosthesis-related, such as poor fit, mechanical irritation, reaction to deposits and poor prosthesis hygiene; socket and implant-related, such as exposure, granuloma, peg-related, socket contraction and environmental allergens; lacrimal-related, such as reduced tear production or outflow obstruction; or eyelid-related, such as MGD, lagophthalmos and lack of mucous membrane or skin. A common condition of ocular discomfort in a long-standing patient with ocular prosthesis is giant papillary conjunctivitis, due to a prolonged mechanical irritation and the immunologic reaction of the ocular surface $[11,12]$. Rokohl et al. documented a relationship between the discharge severity of prosthetic eyewear with conjunctival inflammation, higher cleaning frequency and less hand washing before handling [13]. The role of reduced tear production was documented in previous studies. A reduced tear volume was documented in anophthalmic patients, as demonstrated by a reduced Schirmer's test value, as well as a low tear meniscus height $[14,15]$.

Recent studies have also considered the morphologic changes in meibomian glands related to prosthetic eye patients, demonstrating a larger grade of meibomian gland loss compared with the normal paired eyelids [8,9]. In the present study, a high prevalence of MGD was recognized in the population of patients evaluated. In particular, significant differences were demonstrated between the normal eye and the prosthetic eye, concerning ocular symptoms ( $p$-value $>0.0001$ ), LMA $(p=0.0006)$, meibomian gland expression score $(p=0.0003)$, lid margin abnormalities $(p=0.0006)$ and meiboscore $(p=0.0003)$. Meiboscore was evaluated using Keratograph 5M (Oculus, Wetzlar, Germany) to acquire a noninvasive meibography. MGD describes a group of disorders characterized by functional abnormalities of the meibomian glands. The International Workshop defined MGD a chronic, diffuse abnormality of the meibomian glands, commonly characterized by terminal duct obstruction and/or qualitative/quantitative changes in glandular secretion [16]. MGD can lead to altered tear film composition, ocular surface disease, ocular and eyelid discomfort, and evaporative dry eye. In fact, MGD represents the principal cause of evaporative dry eye [17]. Eyelids of a prosthetic eye seem mostly disposed to obstructive MGD, as result of an increased hyperkeratinization, causing excretory duct obstruction, due to a combination of tear deficiency, deposit accumulation, and micro-trauma. Other hypothetical pathophysiological mechanisms of meibomian gland obstruction, in the eyelid of patients with an ocular prosthesis, is decreased and weakened eyelid blinking. Recently, it was suggested that tear insufficiency as consequent of MGD may be the cause of ocular discomfort for those patients, particularly in whom no specific etiology can be identified. Dry eye symptoms are common in long-standing ocular prosthesis wearers. In this study, a significant difference was demonstrated by comparing the normal eye and prosthetic eye $(p$-value $<0.0001)$ relative to ocular discomfort symptoms. Duration of the prosthesis may play a central role; thus, a positive correlation 
with meiboscore in prosthetic eye was seen $(\mathrm{r}=0.878, p<0.001)$. Jang, et al. reported a greater degree of meibomian gland loss in patients who had used an ocular prosthesis longer than 10 years. In our group of patients, the average duration of prosthesis use was 13.6 \pm 5.1 years. For this reason, MGD could represent an undiagnosed cause of chronic ocular discomfort in long-standing ocular prosthesis patients. The occurrence of biofilm growth has been confirmed on many ocular prostheses. Biofilms may play a significant role in the tolerability of the ocular prosthesis. However, biofilm has also been identified on the surface of scleral implants, without signs of clinical infection. The longstanding ocular prosthesis has been related to the development of giant papillary conjunctivitis, resulting in poor tolerability [18]. Litwin, et al. analyzed the role of the degree of prosthesis surface polishing, comparing the standard and the optical polishing procedures. They reported better tolerability at 12 months in the optical polish of the prosthesis group [19]. Patients with an ocular prosthesis reported a high level of social anxiety, and avoid social situations as a consequence of their altered appearance. This was documented by the mean score $(2.8 \pm 1.13)$ relative to the Facial Appearance subscale of the Negative Physical Self Scale (NPSS). A score $>2$ was observed in eight patients, indicating displeasure with facial appearance. Similar results were also noted relative to the DAS24 questionnaire score. The mean score of DAS24 in our group of patients was $45.88 \pm 25.63$.

DAS24 is a measure of social anxiety and social avoidance in relation to appearance. Sixteen patients had a customized prosthesis, presenting a good aesthetic appearance.

The lowest questionnaire scores were found only in the two patients with stock prostheses.

The HADS is a psychometrical scale assessing patients' anxiety and depression levels related to health problems. Considering the score of the HADS questionnaire, a high level of depression $(6.29 \pm 2.77)$ and anxiety $(6.76 \pm 2.33)$ were detected in our population of patients.

Chronic ocular discomfort could represent a factor that increases the anxiety condition, in patients with an altered appearance. Several studies have shown an association of dry eye disease with depression and anxiety. However, a direct correlation between the severity of dry eye disease and anxiety or depression was not demonstrated [20]. It is plausible to consider an increased risk of developing anxious and depressive disorders in patients with an ocular prosthesis with symptoms of dry eye and MGD. In fact, a higher score of anxiety and depression was recognized in patients presenting symptoms and signs of MGD. In particular, a statistically significant direct correlation was observed between meiboscore and the anxiety HADS score $(\mathrm{r}=0.912 ; p$-value $=0.001)$, as well as with the depression HADS score $(r=0.870 ; p$-value $>0.0001$ (Figure 2)).

Nevertheless, several factors could influence the psychological behavior of patients, and a chronic ocular discomfort could represent a single factor inside a wide variety of causes. In conclusion, this study suggests that anxiety and depression conditions could be common in patients with eye prostheses, in particular in the presence of chronic ocular discomfort caused by MGD. This result should be confirmed in a bigger cohort of patients, to find other factors, such as sex, age, therapy and metabolic differences, that could play a direct influence.

\section{Materials and Methods}

A prospective, multi-centric analysis was conducted on 18 patients (ten male, eight female) with a unilateral ocular prosthesis, between July 2018 and January 2020. The study was conducted in accordance with the tenets of the Declaration of Helsinki. All patients signed an informed consent after a full explanation of all study-related procedures. Inclusion criteria were ability and willingness to participate in the study, unilateral ocular prosthesis worn at least 2 years and subjective symptoms and signs of ocular discomfort such as burning, wetness, foreign body sensation, pain, itching, dryness, tearing and mucous discharge. Patients with inflammation or infection of the socket, or with a poorly fitted prosthesis were excluded from the study. Each patient was questioned about their duration of prosthesis use and the type of ophthalmic surgery (such as evisceration or enucleation) requiring ocular prosthesis. 


\subsection{Symptom Evaluation}

Patients were asked to assign severity using this scale for the following symptoms: burning, wetness, foreign body sensation, pain, itching, dryness, tearing, mucous discharge, hyperemia, excessive blinking, uncomfortable in windy conditions and uncomfortable in dry conditions, evaluated according to a scoring system from 0 (absent) to 3 (severe). A global score was obtained by summing up the scores of each symptom and the values (score range $0-36$ ). The assessment of symptoms was the same for the prosthetic eye-wearing side and for the normal side.

\subsection{Meibomian Gland and Lid Margin Evaluation}

A slit-lamp examination was conducted to evaluate lid margin abnormalities and meibomian gland expression. Lid margin abnormalities were scored from 0 to 4 based on the presence or absence of the following parameters: irregular lid margin, plugging of meibomian gland orifices, vascular engorgement, or a shift in the mucocutaneous junction [10]. Meibomian gland expression was assigned grades for clarity and ease of meibum expression (grade 1-4). Meibography was performed using a Keratograph 5M (Oculus, Wetzlar, Germany) in the upper and lower eyelid separately. Meiboscore grading was assessed by Keratograph 5M automatic software (JENVIS Meibo Grading Scales). Grade 0 : no loss of Meibomian glands; Grade 1: loss of less than 1/3 of the total meibomian gland area; Grade 2: loss of $1 / 3$ to $2 / 3$ of the total area; Grade 3: loss of more than $2 / 3$ of the area. The total Meiboscore was considered as the sum of the meiboscore of the upper eyelid and lower eyelid [21].

\subsection{Psychosocial Variables}

Four standardized questionnaires assessing quality of life, anxiety and depression related to physical health problems, social anxiety and social avoidance in relation to appearance, and three supplementary elements examining the level of shamefulness, sadness and anger felt using a visual analogue scale (0-10). The National Eye Institute Visual Function Questionnaire (NEI VFQ) is a questionnaire assessing the quality of life related to visual function, comprising several subcategories including general and peripheral vision, color perception, ocular discomfort, near and distance activities, social performance, mental healthiness and driving ability. A complex score is estimated by the average of the subcategories' scores (0-100). Lower scores denote poor functioning [22]. The Facial Appearance subscale of the Negative Physical Self Scale (NPSS) is an effective measure of appearance, comprising five categories. We considered the Facial Appearance subscale. The NPSS-F score is estimated by the average of the item scores ( $>2$ indicating displeasure with facial appearance) [23]. The Hospital Anxiety and Depression Scale (HADS) was used to evaluate the level of anxiety and depression. The HADS comprises seven items (scored 0-3) about anxiety, and seven items about depression (scored 0-3). Total scores range from 0 to 21 for both subcategories, with lower scores signifying low levels of anxiety or depression [24]. The DAS24 is a measure of social anxiety and social avoidance in relation to appearance. The total score ranges from 11 to 96 , with high scores signifying high levels of social anxiety and social avoidance [25].

\subsection{Statistical Analysis}

The numerical data were expressed as mean and standard deviation, and the categorical variables as absolute frequency and percentage. Examined variables did not present normal distribution, as verified by the Kolmogorov Smirnov test; consequently, the non-parametric approach was used. For each parameter, we performed statistical comparisons between the normal eye and the prosthetic eye in the exam, using the Wilcoxon signed-rank test for numerical variables. The Pearson correlation coefficient (r) was calculated to measure the strength of correlations between parameters. Statistical analyses were performed using JASP (Version 0.11.1). A $p$-value smaller than 0.05 was considered to be statistically significant. 
Author Contributions: Conceptualization, G.W.O. and A.M.; methodology, G.W.O.; software, G.W.O.; validation, A.M., R.F. and M.R.; formal analysis, G.W.O.; investigation, A.M.; resources, A.M.; data curation, G.W.O.; writing—original draft preparation, G.W.O.; writing—review and editing, A.M.; visualization, R.M.; supervision, A.M.; project administration, A.M.; funding acquisition, A.M. All authors have read and agreed to the published version of the manuscript.

Funding: This research received no external funding.

Conflicts of Interest: The authors declare no conflict of interest.

\section{References}

1. McBain, H.B.; Ezra, D.G.; Rose, G.E.; Newman, S.P.; Appearance Research Collaboration (ARC). The psychosocial impact of living with an ocular prosthesis. Orbit 2013, 33, 39-44. [CrossRef] [PubMed]

2. Kulkarni, R.S.; Kulkarni, P.; Shah, R.J.; Tomar, B. Aesthetically Characterized Ocular Prosthesis. J. Coll. Physicians Surg. Pak. 2018, 28, 476-478. [CrossRef] [PubMed]

3. Modugno, A.; Mantelli, F.; Sposato, S.; Moretti, C.; Lambiase, A.; Bonini, S. Ocular prostheses in the last century: A retrospective analysis of 8018 patients. Eye 2013, 27, 865-870. [CrossRef] [PubMed]

4. Zigiotti, G.L.; Cavarretta, S.; Morara, M.; Nam, S.M.; Ranno, S.; Pichi, F.; Lembo, A.; Lupo, S.; Nucci, P.; Meduri, A. Standard enucleation with aluminium oxide implant (bioceramic) covered with patient's sclera. Sci. World J. 2012, 2012, 481584. [CrossRef]

5. Sajjad, A. Ocular Prosthesis-A Simulation of Human Anatomy: A Literature Review. Cureus 2012, 4, e74. [CrossRef]

6. Jones, C.A.; Collin, J.R. A classification and review the causes of discharging sockets. Trans. Ophthalmol. Soc. UK 1983, 103, 351-353.

7. Malhotra, R. Ocular prostheses: Not quite an eye for an eye. Br. J. Ophthalmol. 2013, 97, 383-385. [CrossRef]

8. Altin, M.E.; Karadeniz, S.U.; Kahraman, H.G. Meibomian Gland Dysfunction and Its Association with Ocular Discomfort in Patients with Ocular Prosthesis. Eye Contact Lens 2019. [CrossRef]

9. Jang, S.Y.; Lee, S.Y.; Yoon, J.S. Meibomian gland dysfunction in longstanding prosthetic eye wearers. Br. J. Ophthalmol. 2013, 97, 398-402, Erratum in 2013, 97, 1362. [CrossRef]

10. Arita, R.; Itoh, K.; Maeda, S.; Maeda, K.; Furuta, A.; Fukuoka, S.; Tomidokoro, A.; Amano, S. Proposed Diagnostic Criteria for Obstructive Meibomian Gland Dysfunction. Ophthalmology 2009, 116, 2058-2063. [CrossRef]

11. Chang, W.J.; Tse, D.T.; Rosa, R.H.; Huang, A.J.W.; Johnson, T.E.; Schiffman, J. Conjunctival Cytology Features of Giant Papillary Conjunctivitis Associated with Ocular Prostheses. Ophthalmic Plast. Reconstr. Surg. 2005, 21, 39-45. [CrossRef] [PubMed]

12. Kim, J.H.; Lee, M.J.; Choung, H.K.; Kim, N.J.; Hwang, S.W.; Sung, M.S.; Khwarg, S.I. Conjunctival cytologic features in anophthalmic patients wearing an ocular prosthesis. Ophthalmic Plast. Reconstr. Surg. 2008, 24, 290-295. [CrossRef]

13. Rokohl, A.C.; Adler, W.; Koch, K.R.; Mor, J.M.; Jia, R.; Trester, M.; Pine, N.S.; Pine, K.R.; Heindl, L.M. Cryolite glass prosthetic eyes-The response of the anophthalmic socket. Graefe's Arch. Clin. Exp. Ophthalmol. 2019, 257, 2015-2023. [CrossRef] [PubMed]

14. Kim, S.E.; Yoon, J.S.; Lee, S.Y. Tear measurement in prosthetic eye users with fourier-domain optical coherence tomography. Am. J. Ophthalmol. 2010, 149, 602-607. [CrossRef] [PubMed]

15. Allen, L.; Kolder, H.E.; Bulgarelli, E.M.; Bulgarelli, D.M. Artificial eyes and tear measurements. Ophthalmology 1980, 87, 155-157. [CrossRef]

16. Nichols, K.K. The international workshop on meibomian gland dysfunction: Introduction. Investig. Ophthalmol. Vis. Sci. 2011, 52, 1917-1921. [CrossRef]

17. Chhadva, P.; Goldhardt, R.; Galor, A. Meibomian Gland Disease: The Role of Gland Dysfunction in Dry Eye Disease. Ophthalmology 2017, 124, S20-S26. [CrossRef]

18. Sun, M.T.; Pirbhai, A.; Selva, D. Bacterial biofilms associated with ocular prostheses. Clin. Exp. Ophthalmol. 2015, 43, 602-603. [CrossRef]

19. Litwin, A.S.; Worrell, E.; Roos, J.C.P.; Edwards, B.; Malhotra, R. Can We Improve the Tolerance of an Ocular Prosthesis by Enhancing Its Surface Finish? Ophthalmic Plast. Reconstr. Surg. 2018, 34, 130-135. [CrossRef] 
20. Kaiser, T.; Janssen, B.; Schrader, S.; Geerling, G. Depressive symptoms, resilience, and personality traits in dry eye disease. Graefe's Arch. Clin. Exp. Ophthalmol. 2019, 257, 591-599. [CrossRef]

21. Pult, H.; Riede-Pult, B. Comparison of subjective grading and objective assessment in meibography. Contact Lens Anterior Eye 2013, 36, 22-27. [CrossRef] [PubMed]

22. Mangione, C.M.; Lee, P.P.; Gutierrez, P.R.; Spritzer, K.; Berry, S.; Hays, R.D. Development of the 25-item National Eye Institute Visual Function Questionnaire. Arch. Ophthalmol. 2001, 119, 1050-1058. [CrossRef]

23. Chen, H.; Jackson, T.; Huang, X. The Negative Physical Self Scale: Initial development and validation in samples of Chinese adolescents and young adults. Body Image 2006, 3, 401-412. [CrossRef] [PubMed]

24. Zigmond, A.S.; Snaith, R.P. The hospital anxiety and depression scale. Acta Psychiatr. Scand. 1983, 67, 361-370. [CrossRef] [PubMed]

25. Carr, T.; Harris, D.; James, C. The Derriford Appearance Scale (DAS-59): A new scale to measure individual responses to living with problems of appearance. Br. J. Health Psychol. 2000, 5, 201-215. [CrossRef]

(C) 2020 by the authors. Licensee MDPI, Basel, Switzerland. This article is an open access article distributed under the terms and conditions of the Creative Commons Attribution (CC BY) license (http://creativecommons.org/licenses/by/4.0/). 